\title{
Mean- Field Approximation and a Small Parameter in Turbulence Theory
}

\author{
Victor Yakhot \\ Department of Aerospace and Mechanical Engineering \\ Boston University, Boston, MA 02215
}

October 30, 2018

\begin{abstract}
Numerical and physical experiments on two-dimensional (2d) turbulence show that the differences of transverse components of velocity field are well described by a gaussian statistics and Kolmogorov scaling exponents. In this case the dissipation fluctuations are irrelevant in the limit of small viscosity. In general, one can assume existence of critical space-dimensionality $d=d_{c}$, at which the energy flux and all odd-order moments of velocity difference change sign and the dissipation fluctuations become dynamically unimportant. At $d<d_{c}$ the flow can be described by the "mean-field theory", leading to the observed gaussian statistics and Kolmogorov scaling of transverse velocity differences. It is shown that in the vicinity of $d=d_{c}$ the ratio of the relaxation and translation characteristic times decreases to zero, thus giving rise to a small parameter of the theory. The expressions for pressure and dissipation contributions to the exact equation for the generating function of transverse velocity differences are derived in the vicinity of $d=d_{c}$. The resulting equation describes experimental data on two-dimensional turbulence and demonstrate onset of intermittency as $d-d_{c}>0$ and $r / L \rightarrow 0$ in three-dimensional flows in close agreement with experimental data. In addition, some new exact relations between correlation functions of velocity differences are derived. It is also predicted that the single-point pdf of transverse velocity difference in developing as well as in the large-scale stabilized two-dimensional turbulence is a gaussian.
\end{abstract}


Mean-Field Approximation and a Small Parameter in Turbulence Theory Victor Yakhot 


\section{Introduction}

The role of the mean-field theories and gaussian limits as starting points for understanding of such important physical phenomena as superconductivity, superfluidity, critical point, naming just a few, can hardly be overestimated. These theories, usually based on remarkable physical intuition and insight, provided mathematical and intellectual basis for investigation of much more difficult regimes in terms of deviations from the mean field solutions. The most recent example is a theory of anomalous scaling in a model of a passive scalar, advected by a random velocity field, which was developed as an expansion in powers of small parameters characterizing deviations from the two gaussian limits [1]-[3]. In a typical non-linear system, a gaussian limit corresponds to a weak coupling asymptotics and, as a consequence, to a "normal", non-anomalous, scaling, which can often be obtained from a "bare' or linearized problem. A good example of this behaviour is a fluid in thermodynamic equilibrium.

The large- Reynolds- number three-dimensional (3d) strong turbulence is characterized by an $O(1)$ energy flux $\mathcal{E}=\nu \overline{\left(\partial_{i} v_{j}\right)^{2}}$, which in many flows is $O\left(v_{r m s}^{3} / L\right)$, where $L$ is an integral scale of turbulence. In the inertial range, where $k_{d}>>k \rightarrow \infty$ or $r / L<<1$, the observed energy spectrum $E(k)$ is close to the one, proposed by Kolmogorov and the probability density $P(\Delta u)$ with $\Delta u=u(x+r)-u(x)$, is far from the gaussian. Moreover, the experiments

revealed that the moments of velocity difference $S_{n, 0}=\overline{(\Delta u)^{n}} \propto r^{\xi_{n}}$ with the exponents $\xi_{n}$ given by very strange ("anomalous") numbers, which cannot be obtained on dimensional grounds. This anomalous scaling and the very existence of the energy flux, resulting in the non-zero value of the third-order moment $S_{3,0}=\overline{(\Delta u)^{3}} \approx O(r)$, where $\mathbf{u} \cdot \mathbf{r}=u r$, implies a strongly non-gaussian process and an obvious lack of the mean-field limit.

The situation may not be so grim, however: all odd-order moments of transverse velocity differences in both $2 d$ and $3 d$ flows $S_{0,2 n+1}=\overline{(v(x+r)-v(x))^{2 n+1}}=0$ with $\mathbf{v} \cdot \mathbf{r}=\mathbf{0}$. This fact tells us that these components of velocity differences do not participate in the inter-scale energy transfer and there is no a'priori any reason for them not to obey gaussian statistics in some limiting cases. This is indeed true in two-dimensional turbulence in the inverse cascade range where $l_{f}<<r<L$ and $l_{f}$ is a forcing scale. 
Numerical and physical experiments on the external- force- driven two- dimensional turbulence showed that the moments of transverse velocity differences and even-order moments of the longitudinal ones are very close to the gaussian values and are characterized by the Kolmogorov scaling exponents $S_{0,2 n} \propto S_{2 n, 0} \propto r^{\xi_{2 n}}$ with $\xi_{2 n}=\frac{2 n}{3}$ [4]-[7]. The odd-order moments of longitudinal velocity differences are positive in a $2 d$ flow, while they all are negative in $3 d$. This observation tells that the most important distinction between two and three-dimensional turbulence in in dynamic role of the dissipation contributions: they are irrelevant in the two-dimensional inverse cascade range and are crucial (see below) for the small-scale dynamics of a three-dimensional flow where the forcing terms can be neglected (see below). Thus, we can assume that it is the dissipation fluctuations that are responsible for both strong deviations from the gaussian statistics and anomalous scaling in three dimensional flows. This assumption is consistent with the observation of the close-to-gaussian probability density of velocity differences in $3 d$ turbulence at the scales $r \approx L$ where the integral scale $L$ is defined as the one at which: $S_{3,0}(L)=0$ and the intermittent dissipation fluctuations disappear [8]. It is clear that this range $(r \approx L)$ is not characterized by a well-defined scaling exponents.

As will become clear below, the forcing contribution to the equation for the probability density involves factor $\mu \approx 1-\cos \left(k_{f} r\right)$. This means that at the scales $r>>1 / k_{f}$ the parameter $\mu \approx 1$ while $\mu \approx\left(k_{f} r\right)^{2}$ when $k_{f} r<<1$. Thus, the forcing term must be important in the inertial range of two-dimensional turbulence $\left(r>>l_{f}\right)$ and is irrelevant in the $3 d$-inertial range with the positive energy flux. This change happens at some space dimensionality $d=d_{c}$ at which the energy flux changes sign. First calculation of $d_{c} \approx 2.05$ was conducted by Frisch et. al. [9] within the framework of a simple closure model. The more physically transparent calculation can be performed for the Navier-Stokes equations driven by a random force having an algebraically decaying spectrum in the inertial range [10], where a one- loop small-scale- elimination procedure gives a correction to the bare viscosity:

$$
\delta \nu=\nu \frac{d^{2}-d-\epsilon}{2 d(d+2)} R e^{2}
$$

where Re is a properly defined Reynolds number corresponding to the eliminated small-scale 
velocity fluctuations and $\epsilon$ is a parameter characterizing the forcing function. In case of Kolmogorov turbulence $\epsilon \approx 4$. This relation shows that the role, the small-scales play in turbulence dynamics, depends on the space dimensionality $d$ : the correction to viscosity is positive when $d>d_{c}(\epsilon)$ where it changes sign. Physically, this means that the small-scale velocity fluctuations take energy from the large- scales motions (direct energy cascade from large-to-small structures) at $d>d_{c}$, while at $d<d_{c}$ they excite the large scale motions (spend their energy) giving rise to the inverse energy cascade. For $\epsilon=4$ the critical dimensionality $d_{c} \approx 2.56$. The correct value of $d_{c}$ is not too important: what is crucial for the theory presented below is that the critical dimensionality, at which the flux changes its sign, exists. It will be shown below that $d-d_{c} \rightarrow 0$ is a small parameter of the theory enabling one to calculate an expression for the dissipation anomaly in the form, resembling the Kolmogorov refined similarity hypothesis.

Since in $2 d$ the moments $S_{0,2 n}$ show Kolmogorov scaling, the gaussian statistics of transverse velocity differences cannot correspond to the weak coupling limit. This problem was considered in the Ref. [11] where, following Polyakov [12], the equation for the generating function for the problem of the Navier-Stokes turbulence was first introduced . An unusual symmetry of this equation enabled one to show that the solution was consistent with both Kolmogorov scaling and gaussian statistics. In this work a more detailed theory of two-dimensional turbulence is presented and the generalization to three-dimensional flows is considered. The main result of the paper is a model demonstrating how the deviations from the "normal scaling" and gaussian statistics appear in $3 d$ when the strength of the dissipation term $\beta>0$ and the "scale" parameter $\epsilon=1-r / L$ deviate from zero.

This paper is organized as follows. In Section 2 the equation for the generating function, derived in [11] is introduced. Some new exact relations between velocity structure functions, following from this equation, are derived in Section 3. The connection between scaling exponents of the moments of velocity differences and their amplitudes is established in Section 4. The mean-field derivation of the pressure term is given in Section 5 which is used to obtain a gaussian pdf in the two-dimensional flow in Section 6. In Sections 7 the small parameter of the theory is identified and used for derivation of the expression for the dissipation con- 
tributions to the eqaution for the pdf. Section 8 is devoted to solution of the equation and demonstration how anomalous scaling and deviations from gaussian statistics emergr from the theory. Conclusive remarks are presented in Section 9.

\section{Equation for the Generating Function}

The equations of motion are (density $\rho \equiv 1)$ :

$$
\partial_{t} v_{i}+v_{j} \partial_{j} v_{i}=-\partial_{i} p+\nu \nabla^{2} v_{i}+f_{i} ; \quad \partial_{i} v_{i}=0
$$

where $\mathbf{f}$ is a forcing function responsible for the large-scale kinetic energy production and in a statistically steady state the mean pumping rate $P=\overline{\mathbf{f} \cdot \mathbf{v}}$. In what follows we will be mainly interested in the probability density function of two-point velocity difference $\mathbf{U}=$ $\mathbf{u}\left(\mathbf{x}^{\prime}\right)-\mathbf{u}(\mathbf{x}) \equiv \Delta \mathbf{u}$. The generating function is: $Z=<\exp (\lambda \cdot \mathbf{U})>$. The equation for the generating function of velocity differences corresponding to (1) is:

$$
\frac{\partial Z}{\partial t}+\frac{\partial^{2} Z}{\partial \lambda_{\mu} \partial r_{\mu}}=I_{f}+I_{p}+D
$$

with

$$
\begin{gathered}
I_{f}=<\lambda \cdot \Delta \mathbf{f} e^{\lambda \cdot \Delta \mathbf{u}}> \\
I_{p}=-\lambda \cdot<e^{\lambda \cdot \Delta \mathbf{u}} \Delta(\nabla p)>\equiv-\lambda \cdot<e^{\lambda \cdot \mathbf{v}}\left(\nabla_{\mathbf{2}} p\left(x_{2}\right)-\nabla_{\mathbf{1}} p\left(x_{1}\right)\right)>
\end{gathered}
$$

and

$$
D=\nu \lambda \cdot<\left(\nabla_{\mathbf{2}}^{\mathbf{2}} \mathbf{v}\left(\mathbf{x}_{\mathbf{2}}\right)-\nabla_{\mathbf{1}}^{\mathbf{2}} \mathbf{v}\left(\mathbf{x}_{\mathbf{1}}\right)\right) e^{\lambda \cdot \mathbf{U}}>
$$

The most interesting and surprising feature of (2) is the fact that, unlike in the problem of Burgers turbulence [12], the advective contributions are represented there in a closed form. This means that the theory, developed below, is free from the troubles related to Galileo invariance, haunting all schemes, based on renormalized perturbation expansions in powers of 
Reynolds number. To completely close the problem the expressions for $I_{p}$ and $D$ are needed. The equations (2)-(3) formulate the turbulence theory in terms of "only" two unknowns $I_{p}$ and $D$. The Kolmogorov refined similarity hypothesis stating that $(\Delta u)^{3}=\phi \mathcal{E}_{r} r$ where $\phi$ is a scale-independent random process and $\mathcal{E}_{r}$ is a dissipation rate averaged over a ball of radius $r$ around point $x$, can be a promising starting point to a closure for the dissipation term $D$. This will be done below. The pressure term in (2)-(3) is also of a very specific and rather limited nature: all we have to know is the correlation functions $<U_{i} U_{j} \cdots U_{m} \Delta \nabla p>$. Thus, the definite targets needed for derivation of the closed equation for $Z$-functions are well-defined.

The generating function can depend only on three variables: $\eta_{1}=r ; \quad \eta_{2}=\frac{\lambda \cdot \mathbf{r}}{r} \equiv$ $\lambda \cos (\theta) ; \quad \eta_{3}=\sqrt{\lambda^{2}-\eta_{2}^{2}} ;$ and

$$
Z_{t}+\left[\partial_{\eta_{1}} \partial_{\eta_{2}}+\frac{d-1}{r} \partial_{\eta_{2}}+\frac{\eta_{3}}{r} \partial_{\eta_{2}} \partial_{\eta_{3}}+\frac{(2-d) \eta_{2}}{r \eta_{3}} \partial_{\eta_{3}}-\frac{\eta_{2}}{r} \partial_{\eta_{3}}^{2}\right] Z=I_{f}+I_{p}+D
$$

where, to simplify notation we set $\partial_{i, \alpha} \equiv \frac{\partial}{\partial x \cdot \alpha}$ and $v(i) \equiv v\left(\mathbf{x}_{\mathbf{i}}\right)$. Below we will often use $\partial_{\eta_{i}} \equiv \partial_{i}$. The functions $I_{p}, I_{f}$ and $D$ are easily extracted from the above definitions. Let us denote $\Delta u \equiv U$ and $\Delta v \equiv V$.

In the new variables the generating function can be represented as:

$$
Z=<e^{\eta_{2} \Delta u+\eta_{3} \Delta v}>\equiv<e^{\eta_{2} U+\eta_{3} V}>
$$

with the mean dissipation rate $\mathcal{E}$ defined by: $\overline{\nu\left(\partial_{x_{i}} u\right)^{2}}=\frac{1}{d} \mathcal{E}$. Any correlation function is thus:

$$
S_{n, m} \equiv<U^{n} V^{m}>=\partial_{2}^{n} \partial_{3}^{m} Z\left(\eta_{2}=\eta_{3}=0, r\right)
$$

\section{Relations between moments of velocity difference}

Let us discuss some direct consequences of equations (1)-(6). The Navier-Stokes equations are invariant under transformation: $v \rightarrow-v$ and $y \rightarrow-y$. That is why: $<\left(\partial_{y} p(0)-\right.$ $\left.\partial_{y} p(r)\right)(\Delta v)^{m}>\neq 0$ if $m=2 n+1$ with $n>1$ and is equal to zero if $m=2 n$. It is also clear 
from the symmetry that $<\partial_{x} p U^{2 n}>=0$. It follows from the Navier-Stokes equations that $<\left(\nabla^{2} U\right) U^{2 n}>=<\left(\nabla^{2} V\right) V^{2 n}>=0$.

Multiplying (6) by $\eta_{3}$, applying $\partial_{2}^{2 n-1}$ to the resulting equation gives as $\eta_{2}=\eta_{3} \rightarrow 0$

$$
\frac{\partial S_{2 n, 0}}{\partial r}+\frac{d-1}{r} S_{2 n, 0}-\frac{(d-1)(2 n-1)}{r} S_{2 n-2,2}=P\left(1-\cos \left(r / l_{f}\right)\right) \frac{2(2 n-1)(2 n-2)}{d} S_{2 n-3,0}
$$

The right side of (7) is $O\left(r^{2}\right)$ in a three-dimensional flow (3d) where $r / l_{f}<<1$ and and can be neglected. It is however is $O(1)$ in two dimensional turbulence in the inverse cascade range where $r / l_{f}>>1$. The dissipation terms do not contribute to this relation. For $n=1$ (7) gives a well-known incompressibility relation [13], [14]:

$$
\frac{\partial S_{2,0}}{\partial r}+\frac{d-1}{r} S_{2,0}=\frac{d-1}{r} S_{0,2}
$$

Multiplying (6) by $\eta_{3}$ with subsequent $\partial_{3} \partial_{2}^{2}$ leads after setting $\eta_{2}=\eta_{3}=0$ as $\nu \rightarrow 0$ to:

$$
\frac{\partial S_{3,0}}{\partial r}+\frac{d-1}{r} S_{3,0}-2 \frac{d-1}{r} S_{1,2}=(-1)^{d} \frac{4}{d} P
$$

where $d=2 ; 3$. Applying $\partial_{3}^{3} \eta_{3}$ to (6) gives, as $\nu \rightarrow 0$ :

$$
\frac{\partial S_{1,2}}{\partial r}+\frac{d+1}{r} S_{1,2}=(-1)^{d} \frac{4}{d} P
$$

Substituting this into (9) yields a well-known Kolmogorov relation:

$$
S_{3,0} \equiv \overline{(\Delta u)^{3}}=(-1)^{d} \frac{12}{d(d+2)} \operatorname{Pr}
$$

For $2 n=4$ the relation $(7)$ reads:

$$
\frac{\partial S_{4,0}}{\partial r}+\frac{d-1}{r} S_{4,0}=\frac{3(d-1)}{r} S_{2,2}
$$

In two-dimensional turbulence $(d=2)$ in the inverse cascade range one can neglect the dissipation contribution $D$ (see below) and derive:

$$
\frac{\partial S_{1,2 n}}{\partial r}+\frac{1+2 n}{r} S_{1,2 n}=n(2 n-1) P S_{0,2 n-2}-2 n<\mathcal{P}_{y v}(\Delta v)^{2 n-1}>
$$


where $\mathcal{P}_{y v} \equiv \partial_{y} v(x+r)-\partial_{y} v(x)$. Another interesting relation, valid in $2 d$ is obtained from (6) readily:

$$
\frac{\partial S_{2,2 n}}{\partial r}+\frac{1+2 n}{r} S_{2,2 n}=\frac{S_{0,2 n+2}}{r}-2 n<\mathcal{P}_{y v} \Delta u(\Delta v)^{2 n-1}>+n(2 n-1) P S_{1,2 n-2}
$$

In the direct cascade range, where the forcing contribution is $O\left(r^{2}\right) \rightarrow 0$, the relation (14) for an arbitrary dimensionality $d$ reads:

$$
\frac{\partial S_{2,2 n}}{\partial r}+\frac{d-1+2 n}{r} S_{2,2 n}=\frac{2 n+d-1}{2 n+1} \frac{S_{0,2 n+2}}{r}-2 n<\mathcal{P}_{y v} \Delta u(\Delta v)^{2 n-1}>
$$

Now, let us multiply (6) by $\eta_{3}$, differentiate once over $\eta_{2}$ and three times over $\eta_{3}$. This gives:

$$
\frac{\partial S_{2,2}}{\partial r}+\frac{d+1}{r} S_{2,2}=\frac{d+1}{3 r} S_{0,4}-2 \overline{\mathcal{P}_{y v} \Delta u \Delta v}
$$

This relation is correct since $\nu \overline{\nabla^{2} v \Delta u \Delta v}=\nu \overline{\nabla^{2} u(\Delta v)^{2}}=0$.

2d simulations of Bofetta, Celani and Vergassola To achieve a true steady state these authors [7] conducted a series of very accurate simulations of the problem (1) with the large-scale dissipation term $D_{L}=-\alpha \mathbf{v}$ in the right side (6). The moments of transverse velocity differences, reported in this paper $(n \geq 2)$, were very close to their gaussian values. It is clear that this term introduces $-\alpha \lambda_{\mu} \frac{\partial Z}{\partial \lambda_{\mu}}$ into the right side of (6) which is small in the inertial range where the non-linearity is large. One has to be careful though with the dangerous interval $\Delta v \rightarrow 0$ where the linear term is not small. We expect the negative- order structure functions with $-1<n<0$ strongly depend on the functional shape of the otherwise irrelevant large-scale dissipation term. The same can be predicted for various conditional expectation values of dynamical variables, like pressure gradients and dissipation terms, for the fixed values of $\Delta v \Delta u$ : near the origin where $\Delta v$ and $\Delta u$ are very small, the artificially introduced linear contributions to the Navier-Stokes equations dominates, producing large and non-universal deviations from the universal functions characterizing inertial range. For example, with addition of the linear dissipation, the relation (14) reads: 
$\frac{\partial S_{2,2 n}}{\partial r}+\frac{1+2 n}{r} S_{2,2 n}=\frac{S_{0,2 n+2}}{r}-(2 n+1) \alpha S_{1,2 n}-2 n<\mathcal{P}_{y v} \Delta u(\Delta v)^{2 n-1}>+n(2 n-1) P S_{1,2 n-2}$

modifying the balance (pressure contribution) in the range of small product $\Delta u \Delta v$ or $r / L \approx$ 1. In the interval where $\Delta u \Delta v$ is not small $(r \rightarrow 0)$, the linear terms are small and can be neglected.

The results obtained in this section can also be derived with

$$
Z=e^{\sqrt{d-1} \eta_{3} V+\eta_{2} U}
$$

with the properly defined moments of $V$ and $U$.

\section{Asymptotic values of exponents in three-dimensional flows}

In case of intermittent turbulence $S_{m, n}=A_{m, n} r^{\xi_{m, n}}$. We can see that in the inertial range of a three-dimensional flow $\left(r / l_{f}<<1\right)$ the right side of $(7)$ is negligible and, as a result, $\xi_{2 n, 0}=\xi_{2 n-2,2} \equiv \xi_{2 n}<\frac{2 n}{3}$. Substituting this into (7) gives immediately:

$$
\xi_{2 n}=(d-1)\left[(2 n-1) \frac{A_{2 n-2,2}}{A_{2 n, 0}}-1\right]
$$

Let us introduce the probability density functions:

$$
S_{2 n, 0}=\overline{U^{2 n}}=\int P(U) U^{2 n} d U
$$

and

$$
S_{2 n-2,2}=\overline{U^{2 n-2} V^{2}}=\int P(U) U^{2 n-2} q(V \mid U) d U d V
$$

where $q(V \mid U)$ is conditional pdf of $V$ for fixed value of $U$. It is clear that $q(V \mid U)=q(-V, U)$, so that all odd-order moments of $V$ are equal to zero.. This expression can be rewritten as:

$$
S_{2 n-2,2}=\overline{U^{2 n-2} V^{2}}=\int P(U) U^{2 n-2} Q_{2}(U) d U
$$


where $Q_{2}$ is a conditional expectation value of $V^{2}$ for a fixed value of $U$ :

$$
Q_{2}(U)=\int V^{2} q(V \mid U) d V
$$

Comparing the above expressions we observe that the amplitudes $A_{2 n, 0} \approx A_{2 n-2,2}$ only if $Q_{2} \propto U^{2}$. This seems rather improbable in the limit $U \rightarrow \infty(n \rightarrow \infty)$. As a result, the linear regime $\xi_{2 n} \propto n$ is equally improbable.

Saturation of exponents $\xi_{2 n} \rightarrow \xi_{\infty}=$ const as $n \rightarrow \infty$ is possible on a rather wide class of probability densities. For example,

$$
P(U) \rightarrow-A \frac{1}{U} \frac{\partial}{\partial U} P(U) Q_{2}(U)
$$

where $A>0$ is a constant. Then,

$$
S_{2 n, 0}=(2 n-1) A \int P(U) Q_{2}(U) U^{2 n-2} d U=(2 n-1) A S_{2 n-2,2}
$$

Substituting this result into the expression for $\xi_{2 n}$ gives $\xi_{2 n} \rightarrow(d-1)(A-1)=$ const. The relation (24) defines the large- $U$ asymptotics of the pdf $P(U)$ in terms of $Q_{2}(U)$ :

$$
P(U) \propto \frac{1}{Q_{2}(U)} e^{-A \int^{U} \frac{u d u}{Q_{2}(u)}}
$$

If $Q_{2}(U) \rightarrow U^{\beta}$, then, assuming the existence of all moments, it follows from (25) that $\beta<2$. The "lognormal " pdf $P(U)$ corresponds to $Q_{2}(U) \propto U^{2} / 2 \log (U)$. The expression (24) also gives in the limit of large $n$ :

$$
S_{2 n+1,0}=\overline{U^{2 n+1}}=2 n A \overline{V^{2} U^{2 n-1}}=2 n A S_{2 n-1,2}
$$

\section{Pressure contributions}

Due to the symmetries of the Navier-Stokes equations, neither pressure nor dissipation terms contributed to the expressions (7) -(12). To proceed further we have to evaluate $I_{p}$ and $D$. 
First of all we see from (12) that $\xi_{4,0}=\xi_{2,2}$. Let us assume that in the inertial range $S_{4,0}=A_{4,0} r^{\xi_{4,0}}, S_{0,4}=A_{0,4} r^{\xi_{0,4}}$ and $S_{2,2}=A_{2,2} r^{\xi_{2,2}}$. Then, it is clear from (12) and (16) that neglecting the pressure contribution to (16) gives $\xi_{4,0}=\xi_{0,4}$.

$\mathbf{d}=\mathbf{2}$. It will be shown below that in $2 \mathrm{~d}$ the even-order moments of velocity differences are very close to the gaussian ones and all exponents are close to the K41 values $\xi_{n}=n / 3$. Then, $A_{4,0}=3 A_{2,0}^{2}$ and $A_{0,4}=3 A_{0,2}^{2}$. It follows from (12) that $A_{2,2}=\frac{7}{9} A_{4,0}$. Taking into account that when $d=2$ the amplitudes $\frac{5}{3} A_{2,0}=A_{0,2}$, we conclude that without the pressure contribution the equations (12) and (16) are incompatible.

Following [15] we introduce a conditional expectation value of the pressure gradient difference for a fixed value of $\Delta u, \Delta v$ and $r$ :

$$
<\partial_{y} p(x+r)-\partial_{y} p(x) \mid \Delta u, \Delta v, r>\approx \sum_{m, n} \kappa_{m, n}(r)(\Delta u)^{m}(\Delta v)^{n}
$$

where the functions $\kappa_{m, n}(r)$ ensure proper dimensionality of the corresponding correlation functions. The above expression explicitly assumes existence of an expansion of conditional expectation value (28). In general, this may not be true due to various singularities such as the ones arising in the dissipation contributions (see below). Since the pressure term involves only one spacial derivative, the ultra-violet singularity cannot appear. The infrared singularity is not there at least in $2 \mathrm{~d}$ where the integral scale is time-dependent (see below). Keeping only the first two terms of the expansion (28), produces a model for the pressure contributions:

$$
<\partial_{y} p(x+r)-\partial_{y} p(x) \mid \Delta y \Delta v>\approx-h \frac{\Delta u \Delta v}{r}-b \frac{\Delta v}{(\operatorname{Pr})^{\frac{2}{3}}}
$$

Since in an incompressible and homogeneous flow $\overline{\Delta u \mathcal{P}_{x u}}=\overline{\Delta v \mathcal{P}_{y v}}=0$, the coefficients $h$ and $b$ are related as:

$$
-h S_{1,2}=b S_{0,2}(P r)^{\frac{1}{3}}
$$

Limiting the expansion of a conditional expectation value by the first terms resembles Landau's theory of critical phenomena, well describing experimental data in a certain range of 
parameters variation. We will show below that in case of turbulence this approximation gives the results which are in agreement with the data. This may be a consequence of the fact that $A_{0,4} \approx A_{4,0} \approx A_{2,2}=O(1)$.

With $\xi_{n}=n / 3$ it follows from (7), (13)-(15) and (29) that when $d=2$ and $n \rightarrow \infty$ :

$$
\left(\frac{2 n(4-3 h)}{3}+1\right) \frac{S_{1,2 n}}{r}=n(2 n-1) P S_{0,2 n-2}+b \frac{2 n S_{0,2 n}}{(P r)^{\frac{2}{3}}}
$$

and

$$
\left(\frac{2 n(4-3 h)}{3}+1\right) \frac{S_{2,2 n}}{r}=\frac{S_{0,2 n+2}}{r}+n(2 n-1) P S_{1,2 n-2}+b \frac{2 n S_{1,2 n}}{(\operatorname{Pr})^{\frac{2}{3}}}
$$

In the limit $n \rightarrow \infty$ assuming that $S_{1,2 n} \approx n S_{1,2 n-2}$ one derives readily:

$$
\operatorname{Prn} S_{0,2 n-2} \approx \frac{S_{2,2 n+2}}{\operatorname{Prn}} \approx \frac{1}{\operatorname{Prn}^{2}} S_{0,2 n+4}
$$

which is consistent with the gaussian pdf $P(\Delta v)$ as $\Delta v \rightarrow \infty$. Thus, the relation (29) implies the gaussian tails of the probability density. It is clear that due to the finite energy flux and relations (10)-(11), two-dimensional turbulence cannot be a gaussian process. All the relation (31) can tell us is that the even-order moments with $n>>1$, described by (31), can be close to the gaussian values. It will be shown below that transverse velocity differences, not directly involved in the inter-scale energy transfer, can obey gaussian statistics.

It is clear from (17) that the model (29) for the pressure contributions is wrong when the linear dissipation terms are added to the Navier-Stokes equations. In the limit of small $\Delta u \Delta v$ the balance is achieved when:

$$
<\mathcal{P}_{y v} \mid \Delta u, \Delta v, r>\approx-h \frac{\Delta u \Delta v}{r}-\left(\alpha+\frac{b}{(\operatorname{Pr})^{\frac{2}{3}}}\right) \Delta v
$$

which differs from (29) in the range of small $\Delta u \Delta v$.

3d. In the intermittent three-dimensional turbulence $\xi_{2 n}<\xi_{2 n+1}$. This produces strong restrictions on the structure of the pressure contributions to the equation (6). Let us assume that $\xi_{2 n, 0}=\xi_{2,2 n-2}=\xi_{2 n-2,2}$. Then, it is clear from (15) that the first term of expansion (28) has all right properties. The relation (15), involving the $r$-derivatives, is valid for an arbitrary 
value of $n$ and that is why any additional term of expansion (28) must not only depend on a proper power of $n$ but the functions $\kappa_{m, n}(r)$ must also reflect non-trivial dimensionalities caused by the anomalous scaling exponents $\xi_{n}$. It cannot be rigorously ruled out, though this possibility seems quite bizzarre. In what follows we will adopt the pressure model (29) in the three-dimensional case also.

\section{Two-dimensional turbulence}

Now we will be interested in the case of the two-dimensional turbulence in the inverse cascade range. If a two-dimensional (2d) fluid is stirred by a random (or non-random) forcing, acting

on a scale $l_{f}=1 / k_{f}$, the produced energy is spent on creation of the large-scale $\left(l>l_{f}\right)$ flow which cannot be dissipated in the limit of large Reynolds number as $\nu \rightarrow 0$. This is a direct and most important consequence of an additional, enstrophy conservation, law, characteristic of two dimensional hydrodynamics [16]. As a result, the dissipation terms are irrelevant in the inverse cascade range and we set $D=0$ in (6) and hope that in two dimensions the situation is greatly simplified. This hope is supported by recent numerical and physical experiments [4]-[7] showing that as long as the integral scale $L_{i} \propto t^{\frac{3}{2}}$ is much smaller than the size of the system, the velocity field at the scales $L_{i}>l>>l_{f}$ is a stationary close-togaussian process characterized by the structure functions with the Kolmogorov exponents $\xi_{n}=n / 3$. In a recent paper Bofetta, Celani and Vegrassola [7] reported the results of very accurate numerical simulations of two-dimensional turbulence generated by a random force. No deviations from gaussian statistics of transverse velocity differences as well as from the Kolmogorov scaling $\xi_{n}=n / 3$ were detected. and is equal to zero if $m=2 n$.

The pressure gradient $\partial_{y} p=\partial_{y} \partial_{i} \partial_{j} \partial^{-2} \Delta v_{i} \Delta v_{j}$ and the difficulty in calculation of $I_{p}$ is in the integral over the entire space defined by the inverse Laplacian $\partial^{-2}$. The huge simplification, valid in $2 \mathrm{~d}$, comes from the fact that all contributions to the left side of equation (6) as well as $I_{f}$ are independent on time. This means that the integrals involved in the pressure terms cannot be infra-red divergent since in a two-dimensional flow $L=L(t) \propto t^{\frac{3}{2}}$. We also have that $i_{p} \rightarrow \alpha^{2} i_{p}$ when $U, V \rightarrow \alpha U ; \alpha V$. Based on this and taking into account that 
$<(\Delta v)^{2 n+1}\left(u_{x}^{2}+v_{y}^{2}+u_{y} v_{x}\right)>=0$, we, in the limit $\eta_{2} \rightarrow 0$, adopt a low-order model (29) giving:

$$
I_{p}=\left[h \frac{\partial^{2}}{\partial \eta_{2} \partial \eta_{3}}+b \frac{\eta_{3}}{(P r)^{\frac{2}{3}}} \partial_{3}\right] Z\left(\eta_{2}=0, \eta_{3}, r\right)
$$

In two dimensions the relation (32) combined with (6) in the limit $\eta_{2} \rightarrow 0$, solves the problem 2d turbulence.

Substituting (32), (29) into (6) and, based on (9)-(11), seeking a solution as $\eta_{2} \rightarrow 0$ as

$Z\left(\eta_{2}, \eta_{3}, r\right) \approx Z_{3}\left(\eta_{3}, r\right) \varphi\left(\eta_{2} r^{\frac{1}{3}}, \eta_{2}\right) \approx Z_{3}\left(\eta_{3}, r\right) \exp \left(\frac{1}{2} A_{2,0}\left(\eta_{2} \operatorname{Pr}^{\frac{1}{3}}\right)^{2}\right)\left(1+\frac{1}{2} A_{1,2} \eta_{3}^{2} \eta_{2}(\operatorname{Pr})+\frac{\eta_{2}^{3}}{4}+\ldots\right)$

where $\left(A_{1,2}=1 / 2\right)$, gives:

$$
\left[\partial_{r}+\frac{1}{r}+\frac{1-h}{r} \eta_{3} \partial_{3}\right] \frac{A_{1,2} P r}{2} \eta_{3}^{2} Z_{3}=2 P \eta_{3}^{2} Z_{3}+\frac{b \eta_{3}}{r^{\frac{2}{3}}} \partial_{3} Z_{3}
$$

Setting $Z_{3}=Z_{3}\left(\eta_{3} r^{\frac{1}{3}}\right) \equiv Z_{3}(X)$ and $h=4 / 3$ one derives using, the relation $(30)\left(b=-\frac{2}{3 A_{0,2}}\right)$ :

$$
2 A_{0,2} X Z_{3}=\partial_{X} Z_{3}
$$

corresponding to a Gaussian solution with the correct width $A_{0,2}$. This fact serves as a consistency check that the gaussian is a solution for the PDF of transverse velocity differences. The equation (34) defines a probability density function corresponding to the finite moments $S_{2 m, 2 n}(r)$ only when $h=4 / 3$. This situation resembles Polyakov's theory of Burgers turbulence [12] reduced to an eigenvalue problem with a single eigenvalue corresponding to the pdf which is positive in the entire interval.

Having these exact results, and keeping in mind (33) one can integrate the equation over $\eta_{2}$ from $-i \infty$ to 0 to obtain :

$$
\frac{\partial Z_{3}}{\partial r}+3(1-h-b) \frac{\eta_{3}}{r} \frac{\partial Z_{3}}{\partial \eta_{3}}=\frac{2 P}{(P r)^{\frac{1}{3}}} \eta_{3}^{2} Z_{3}
$$

valid as long as 


$$
\frac{\left(\eta_{3} r^{\frac{1}{3}}\right)^{2}}{8 A_{2,0}^{2}}<<1
$$

This constraint is an artifact of an approximate relation (33). As will be shown below (35) gives an exact gaussian solution and thus, is valid beyond above interval. This result is obtained choosing the integration function $\Psi\left(\eta_{3}, r\right)$ to compensate the $O(Z / r)$ term violating the normalizability constraint $Z(0,0, r)=1$. Solution to (35) is:

$$
Z_{3}=\exp \left(\gamma \eta_{3}^{2}(P r)^{\frac{2}{3}}\right)
$$

with the parameter $\gamma=\frac{3}{(3(1-h-b)+1)} \propto A_{0,2}$ defining the width of the gaussian.

The first-order differential equation (35) for the generating function differences implies the underlying linear Langevin dynamics of transverse velocity differences. It is important that this equation in non-local in physical space but local in the Fourier one. The effective forcing, corresponding to the right-side of (35), is a non-local and solution- dependent.

To evaluate the single-point probability density, corresponding to velocity differences in the limit of large displacements $r$, we can notice that the energy flux is not equal to zero only at $r<<L$. At the distances $r \geq L(t)$ the zero value of the energy flux and symmetrization of the probability density $(P(\Delta u, L)=P(-\Delta u, L)$ can be achieved only when the pressure contribution to the equation (6) compensates the advective terms. As a result, since $D=0$, we have:

$$
Z_{t}=2 P \eta_{3}^{2} Z
$$

Seeking a solution as $Z=Z\left(\eta_{3} \sqrt{t}\right) \equiv Z\left(\eta_{3} v_{r m s}(t)\right)$ gives a gaussian result:

$$
Z=e^{\eta_{3}^{2} u_{r m s}^{2}(t)}
$$

Similar outcome is obtained for the case investigated in [7]. When turbulence is stabilized at the large scales by an artificially introduced friction, the resulting equation is:

$$
\eta_{3} \partial_{3} Z=2 p \eta_{3}^{2} Z
$$


also leading to the gaussian pdf. To conclude this section we would like to discuss the physical meaning of the integral scale $L$. The integral scale of turbulence is a scale at which the flux decreases to zero [18] and at which $S_{3,0}(L)=0$.

\section{$7 \quad$ Small Parameter in Turbulence Theory in Three Dimensions}

Three-dimensional turbulence is a notoriously difficult problem due to absence of the small parameter. This can be illustrated on an example of a coarse-graining procedure, which was extremely successful in engineering turbulence simulations. Consider the wave-number $k$ in

the inertial range. Let us denote $\mathbf{v}^{<}(\mathbf{k})$ the Fourier components of the velocity field with all modes $\mathbf{v}^{>}(\mathbf{q})=0$ when $q>k$. The coarse-grained field in the physical space is defined then:

$$
\mathbf{v}_{r}(\mathbf{x}, t)=\int_{k<\frac{1}{r}} \mathbf{v}^{<}(\mathbf{k}) e^{i \mathbf{k} \cdot \mathbf{x}} d^{3} k
$$

The equation of motion for $v_{i}^{<}(k)$ in the Fourier space resembles the Navier-Stokes equation with effective viscosity

$$
\nu(k) \approx\left(\frac{\left(d-d_{c}\right)\left(d+\frac{1}{2}\right)}{3.2 d(d+2)}\right)^{\frac{1}{3}} \mathcal{E}^{\frac{1}{3}} k^{-\frac{4}{3}}
$$

with $d_{c} \approx 2.56$ plus high order non-linearities. The parameter 3.2 in the above relation, evaluated at $d=3$, is in fact a weak function of space dimensionality and $\mathcal{E}=P=O(1)$. This expression is derived assuming a close-to-gaussian statistics of the small-scale turbulence with

$$
\overline{v_{i}(k, \omega) v_{j}\left(k^{\prime}, \omega^{\prime}\right)} \propto \frac{k^{-d}}{-i \omega+\nu(k) k^{2}} \delta\left(k+k^{\prime}\right) \delta\left(\omega+\omega^{\prime}\right)
$$

which is accurate at $d-d_{c} \rightarrow 0$ [10] (see below).

In the physical space the effective viscosity of the coarse-grained field:

$$
\nu_{r} \approx\left(d-d_{c}\right)^{\frac{1}{3}} N\left(\mathcal{E} r^{4}\right)^{\frac{1}{3}} \approx v_{r}^{2} \tau_{r}
$$


defines the relaxation time $\tau_{r}$ which is a characteristic time of interaction of the field $v_{r}$ with the eliminated modes acting on the scales $l<r$.

The difficulty of the theory is in the higher non-linearities

$$
\left(v_{r} \nabla\right)^{n} \tau_{r}^{n-1} v_{r}
$$

and the dimensinless expansion parameter

$$
\frac{\tau_{r}}{\theta_{r}} \approx \nabla_{r} v_{r} \tau_{r} \approx \frac{\tau_{r} v_{r}}{r}
$$

is nothing but the ratio of translational time $\theta_{r}$, characterizing the tendency of the "largescale" longitudinal velocity fluctuation at the scale $r$ to form a small-scale structure ("shock") in the absence of pressure, to the relaxation time strongly influenced by the pressure gradients contributions. In both $2 \mathrm{~d}$ and $3 \mathrm{~d}$ these times are of the same order and that is why trancation of the expansion is a very difficult problem.

This is not so in the vicinity of $d=d_{c}$. Let us assume that the theory can be analytically continued to the non-integer dimensions [9]. Then, since the energy flux is $O(1)$ we have:

$$
\mathcal{E} \approx \frac{\partial}{\partial r}<\Delta u(\Delta v)^{2}>\approx \frac{\partial}{\partial r}<(\Delta u)^{3}>\approx \nu<\left(\frac{\partial v_{r i}}{\partial r_{j}}\right)^{2}>=O(1)
$$

This means that

$$
v_{r, r m s} \approx\left(d-d_{c}\right)^{-\frac{1}{6}}(\mathcal{E} r)^{\frac{1}{3}}
$$

the dissipation wave-number $k_{d} \approx\left(d-d_{c}\right)^{\frac{1}{4}}\left(\frac{\mathcal{E}}{\nu_{0}^{3}}\right)^{\frac{1}{4}}$ and, as a consequence,

$$
\frac{\tau_{r}}{\theta_{r}} \approx\left(d-d_{c}\right)^{\frac{1}{2}}
$$

which will serve as a small parameter of the theory when $d-d_{c} \rightarrow 0+$.

These relations tell us that the turbulent intensity grows to infinity with $d-d_{c} \rightarrow 0$ where the energy flux changes its sign. The time needed to reach the steady stae is estimated easily: 


$$
T \approx\left(d-d_{c}\right)^{-\frac{1}{3}} \mathcal{E}^{-\frac{1}{3}} r^{\frac{2}{3}}
$$

after which a close-to-Kolmogorov spectrum is expected both above and below $d_{c}$. Thus, at $d=d_{c}$ the the flow is unsteady.

The above results enable one to derive a plausable estimate for the dissipation term $D$. It is clear from the Navier-Stokes equations that:

$$
\mathcal{E}=-\frac{1}{2} \partial_{i} v_{i} v_{j}^{2}-\frac{1}{2} v_{t}^{2}-\partial_{i} v_{i} p
$$

The coarsed-grained expression in the law frequency limit

$$
\mathcal{E} \approx-\frac{1}{2} \frac{\partial}{\partial r_{r}} v_{r i} v_{r j}^{2}\left(1+O\left(d-d_{c}\right)\right)
$$

To arrive in the expression for $D$ we assume $v_{r} \approx \Delta v$ leading to an expression very similar to Kolmogorov's refined similarity hypothesis.

\section{Three-Dimensional Flow}

The most important feature of two-dimensional turbulence, considered in a previous section, is irrelevance of the dissipation processes in the inverse cascade range when $d<d_{c}$. It is this irrelevance which was responsible for the gaussian probability density of transverse velocity differences.

The model for the dissipation contribution $D$ in the limit $\eta_{2} \rightarrow 0$ is evaluated from (37) readily. We would like to keep at least some information about $\Delta v$ and the expression must be invariant under transformation $\mathbf{v} \rightarrow-\mathbf{v}$ and $\mathbf{x} \rightarrow-\mathbf{x}$. In addition, the expression must be local in physical space. Based on this considerations:

$$
\mathcal{E}_{v} \approx c(d) \Delta u \Delta v \frac{\partial \Delta v}{\partial r}
$$

where $\mathcal{E}_{v}$ is a dissipation rate of the "v-component to kitenic energy" $K_{v}=\frac{1}{2} v^{2}$. Locality of this model is clear since $\partial_{r} \Delta v=\partial_{1} v\left(x_{1}\right)+\partial_{2} v\left(x_{2}\right)$. The problem is in evaluation of the 
coefficient $c(d)$ since, in principle, it can be singular at $d=d_{c}$. Indeed, it is clear that the dissipation term is zero at $d \leq d_{c}$. However, the point $d=d_{c}$ separating inverse and direct cascade ranges, is a singularity due to infinitly large amplitudes of velocity fluctuations. All we can say at this point is that the pdf can be represented as a sum of even and odd functions of $\Delta v$. The symmetric part has a growing with $d-d_{c} \rightarrow 0+$ width, while the width of odd one is $O(1)$. The behaviour of $c(d)$ in the vicinity of $d_{c}$ is not clear. We feel that it is $O(1)$ at $d>d_{c}$ and zero at $d \leq d_{c}$.

Thus, we have:

$$
D \approx c(d) \eta_{3} \partial_{\eta_{2}} \partial_{\eta_{3}} \partial_{r} Z \approx c(d) \eta_{3}^{2}<\Delta u \Delta v \frac{\partial \Delta v}{\partial r} e^{\eta_{2} \Delta u+\eta_{3} \Delta v}>+O\left(\partial_{r} \partial_{\eta_{2}} Z\right)
$$

This expression obeys the basic symmetries of the Navier-Stokes equation. The expression, similar to (38), was being used in engineering turbulent modelling based on the low-order in the Reynolds number coarse-grained equations.

The last term in the right side of (38), simply modifies the coefficient in front of the first

term in the left side the equation (6) and does not generate anything new. The expression (38) resembles Kolmogorov's refined similarity hypothesis, connecting the dissipation rate, averaged over a region of radius $r$, with $(\Delta u)^{3}$. Thus, in the limit $\eta_{2} \rightarrow 0$ :

$\left[\partial_{\eta_{1}} \partial_{\eta_{2}}+\frac{2}{r} \partial_{\eta_{2}}+(1+h) \frac{\eta_{3}}{r} \frac{\partial^{2}}{\partial_{\eta_{2}} \partial \eta_{3}}+c(d) \eta_{3} \partial_{\eta_{2}} \partial_{\eta_{3}} \partial_{r}\right] Z\left(\eta_{2}=0, \eta_{3}, r\right)=\frac{2 P}{3}\left(1-\cos \left(k_{f} r\right)\right) Z-b \frac{\eta_{3}}{r^{\frac{2}{3}}} \partial_{3} Z$

The $d-d_{c}>0$ counterpart of equation (35) is:

$$
\left[\partial_{\eta_{1}}+(1+h+b) \frac{\eta_{3}}{r} \frac{\partial}{\partial \eta_{3}}+c(d) \eta_{3} \partial_{\eta_{3}} \partial_{r}\right] Z\left(\eta_{2}=0, \eta_{3}, r\right)=a \frac{2 P}{3} \frac{1-\cos \left(k_{f} r\right)}{r^{\frac{1}{3}}} \eta_{3}^{2} Z_{3}
$$

with $\Psi\left(\eta_{3}\right)$ chosen in such a way that the generating function $Z(0,0, r)=1$. We consider two limiting cases.

\section{Small - ScaleDynamics.}

Inverse Laplace transform of (40) without right side gives an equation for the pdf $P(\Delta v, r)$ : 


$$
\frac{\partial P}{\partial r}+\frac{1+3 \beta}{3 r} \frac{\partial}{\partial V} V P-\beta \frac{\partial}{\partial V} V \frac{\partial P}{\partial r}=0
$$

where $\beta \propto c(d)$. Since $S_{0,3}=0$, the coefficients in (41) are chosen to give $s_{0,3}=\overline{|\Delta v|^{3}}=a_{3} \operatorname{Pr}$ with an undetermined amplitude $a_{3}$. This is an assumption of the present theory, not based on rigorous theoretical considerations. Seeking a solution in a form $S_{0, n}=<(\Delta v)^{n}>\propto r^{\xi_{n}}$ gives:

$$
\xi_{n}=\frac{1+3 \beta}{3(1+\beta n)} n \approx \frac{1.15}{3(1+0.05 n)} n
$$

which was derived in [18] together with $\beta \approx 0.05$. It follows from (42) that: $P(0, r) \propto r^{-\kappa}$ where $\kappa=\frac{1+3 \beta}{3(1-\beta)} \approx 0.4$ for $\beta=0.05$. Very often the experimental data are presented as $P(X, r)$ where $X=V / r^{\mu}$ with $2 \mu=\xi_{2} \approx 0.696$ for $\beta=0.05$. This gives $P(X=0, r) \propto$ $r^{-\kappa+\mu} \approx r^{-0.052}$ compared with the experimental data by Sreenivassan [19]: $-\kappa+\mu \approx-0.06$.

Let us write $P(V, r)=r^{-\kappa} F\left(\frac{V}{r^{\kappa}}, r\right)=r^{-\kappa} F(Y, r)$, so that $F$ obeys the following equation:

$$
(1-\beta) r \frac{\partial F}{\partial r}+\beta \kappa \frac{\partial}{\partial Y} Y^{2} \frac{\partial F}{\partial Y}-\beta Y r \frac{\partial^{2} F}{\partial Y \partial r}=0
$$

Next, changing the variables again $-\infty<y=\operatorname{Ln}(Y)<\infty$, substituting this into (43) and evaluating the Fourier transform of the resulting equation gives:

$$
(1-\beta) r \frac{\partial F}{\partial r}+\beta \kappa\left(i k-k^{2}\right) F-i k \beta r \frac{\partial F}{\partial r}=0
$$

with the result: $F \propto r^{\gamma(k)}$, where $\gamma(k)=\beta \kappa \frac{-i k+k^{2}}{1-\beta-i \beta k} \operatorname{Ln}(r / L)$ with $r / L<<1$. We have to evaluate the inverse Fourier transform:

$$
F=\int_{-\infty}^{\infty} d k e^{-i k y} e^{\gamma(k)}
$$

in the limit $y=O(1)$ and $r \rightarrow 0$ so that $\operatorname{Ln}(r / L) \rightarrow-\infty$. The integral can be calculated exactly. However, the resulting expression is very involved. Expanding the denominator of $\gamma(k)$ gives :

$$
F=\int_{-\infty}^{\infty} d k e^{-i k\left(y+\frac{\beta \kappa(\operatorname{Ln}(r))}{1-\beta}\right)} e^{-\frac{\beta \kappa(1+\beta)|\operatorname{Ln}(r)|}{(1-\beta)} k^{2}}
$$


and

$$
F \propto \frac{1}{\sqrt{\Omega(r)}} \exp \left(-\frac{(\operatorname{Ln}(\xi))^{2}}{4 \Omega}\right)
$$

with $\xi=V / r^{\frac{\kappa}{1-\beta}}$ and $\Omega(r)=4 \beta \kappa \frac{1+\beta}{1-\beta}|\operatorname{Ln}(r / L)|$.

To understand the range of validity of this expression, let us evaluate $\left\langle V^{n}\right\rangle$ using the expression (47) for the pdf. Simple integration, neglecting $O\left(\beta^{2}\right)$ contributions, gives: < $V^{n}>\propto r^{\alpha_{n}}$ with $\alpha_{n}=(1+3 \beta)\left(n-\beta\left(n^{2}+2\right)\right) / 3$. Comparing this relation with the exact result (43) we conclude that the expression for the pdf, calculated above, is valid in the range $n>>1$ and $\beta n<<1$. The properties of the pdf in the range $3 \leq \xi \leq 15$ are demonstrated on Fig. 1 for $r / L=0.1 ; 0.01 ; 0.001$. The log-normal distribution (48), is valid in a certain (wide but limited) range of the $V$ - variation. It is clear from (42) that neglecting the dissipation terms $\left(c\left(d-d_{c}\right) \propto \beta=0\right)$ leads to $\xi_{n}=n / 3$, i. e. disappearance of anomalous scaling of moments of velocity differences. This result agrees with the well-developed phenomenology, attributing intermittency to the dissipation rate fluctuations: the stronger the fluctuations, the smaller the fraction of the total space they occupy [8], [14]. To the best of our knowledge, this is the first work leading to multifractal distribution of velocity differences as a result of approximations made directly on the Navier-Stokes equations.

To investigate the probability density $P(Y, r)$ in the limit $Y \rightarrow 0$ we introduce an expansion:

$$
F(Y, r)=\sum_{n} C_{n} Y^{2 n} f_{2 n}(r)
$$

Substituting this into (44) gives:

$$
f_{2 n} \propto\left(\frac{r}{L}\right)^{-\frac{\beta \kappa 2 n(2 n-1)}{1-\beta(1+n)}}
$$

It is seen from (48)-(49) that the pdf starts bending from the log-normal slope (47) toward $\partial_{Y} F(Y, r)=0$ at $Y=0$ at:

$$
Y<\left(\frac{r}{L}\right)^{\frac{\beta \kappa}{1-2 \beta}}
$$


This inequality shows that as $r \rightarrow 0$ the pdf develops a narrow cusp at the origin $Y=0$. If the probability density is plotted in the dimensionless variable $X$, the bending starts at $X \approx r^{0.07}$. This value was calculated, as above, with $\beta=0.05$.

Large-scale limit: $r / L \approx 1$. Now, let us investigate the large-scale limit $\frac{r}{L} \rightarrow 1$. Realizing that $\beta$ can be an $O(1)$ constant we, for the illustration purposes, will investigate the large scale limit pretending that $\beta(d) \rightarrow 0$ as $d \rightarrow d_{c}$. This is also useful since the estimated value of $\beta \approx 0.05$ at $d=3$ which is numerically small. In this limit the right side of $(41)$ is $O\left(\eta_{3}^{2} Z\right)$ and cannot be neglected. Repeating the procedure leads to an equation:

$$
\frac{\partial P}{\partial r}+\frac{1+3 \beta}{3 r} \frac{\partial}{\partial V} V P-\beta \frac{\partial}{\partial V} V \frac{\partial P}{\partial r}=a \frac{P}{(\operatorname{Pr})^{\frac{1}{3}}} \frac{\partial^{2} Z}{\partial V^{2}}
$$

where $a$ is a proportionality coefficient and $r \approx L$. As one can see from this equation in the limit of small $\beta$ the solution to this equation approaches gaussian. It is also clear that for any finite $\beta$, the tails of the pdf are strongly non-gaussian when

$$
\beta Y^{2}>>1
$$

This estimate means that, according to the theory presented above, the perturbative treatment of deviations from the mean-field gaussian theory is possible but it involves two pa-

rameters: the ratio $\epsilon=1-\frac{r}{L}<<1$ and $\beta<<1$. The fact that the "real-life" $\beta \approx 1 / 20$ may explain why the experimentally observed pdf of the large-scale $(\epsilon<<1)$ velocity fluctuations was so close to the gaussian [see [8] and references therein].

It is also seen from $(52)$ that at $\left(\frac{r}{L}\right)^{2} \approx \beta \approx 0.05$ the pdf is dominated by a gaussian central part.

\section{Conclusions}

The equation (6) formulates theory of turbulence in terms of "only" two unknowns: pressure and dissipation terms $I_{p}$ and $D$, respectively. It provides a mathematical testing ground for various analytic expressions and models obtained from numerical simulations. 
Armed with the experimental and numerical data, supporting gaussian statistics of transverse velocity differenced in two-dimensional flows, we showed that the mean field approximation (the lowest-order term of the expansion (28)) for the pressure contributions (29) leads to both Kolmogorov scaling and gaussian statistics of transverse velocity differences. In addition, the equation (6) shows that the single-point pdf's in $2 d$ turbulence are gaussian. It is to be stressed that $2 d$ turbulence cannot be a gaussian process and probability density $P(\Delta u, \Delta v, r)=$ is not a gaussian. It is only pdf $P(\Delta v, r)=\int P(\Delta u, \Delta v, r) d \Delta u$ is a gaussian. This statement violates no dynamic constraints.

One of the most interesting outcomes of the present theory is a discovery of existence of the two time-scales in the system which are very different in the vicinity of $d=d_{c}$. This difference enables one to coarse-grain the Navier-Stokes equations and neglect all high-order non-linearities, generated by the procedure. Using this result the model for for the dissipation term $D$ was derived.

The as yet unresolved ambiguity of this model is its behaviour as $d \rightarrow d_{c}$. If transition from $3 \mathrm{~d}$ to the non-intermittent state at $d<d_{c}$ is smooth, then $\beta \rightarrow 0$ and the resulting equation shows onset of both anomalous scaling and non-gaussian statistics. The transition can be singular, however: right at $d>d_{c}$ the coefficient $\beta$ can become $O(1)$ and a weakly intermittent state and weak coupling limit do not exist. In this case, due to existence of the small parameter, enabling evaluation of the dissipation expression $D$, the theory nonperturbatively predicts both the shape of the pdf and scaling exponents provided the small parameter $S_{3,0} /\left(S_{2,0}\right)^{\frac{3}{2}} \rightarrow 0$ as $d \rightarrow d_{c}$. This result is possible since $D=D_{0}+O\left(d-d_{c}\right)$ avd even in the limit $d \rightarrow d_{c}$, the model $D=O(1)$. At $d-d_{c}<0, D=0$ leading to the gaussian pdf of transverse velocity differences. Experimental investigation of hydrodynamics in a non-integer space dimension is impossible. However, it was demonstarted by Jensen [20] that a force-driven shell model is yields the changing sign of the energy flux upon variation of a leading parameter. Numerical solution at a critical point (zero flux) demonstrated an unsteady state with the growing total energy and the energy spectrum concentrated in the vicinity of $k_{f}$. The calculat ion also gave kolmogorov energy spectrum at " $d>d_{c}^{\prime \prime}$ with growing Kolmogorov constant as $d \rightarrow d_{c}$. It is not yet clear how the eddy viscosity 


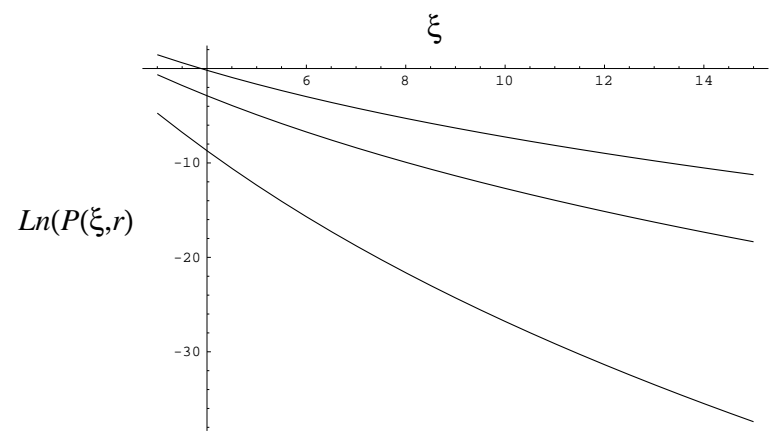

Figure 1: $\operatorname{Ln}(P(\xi, r))$. From bottom to top: $\mathrm{r} / \mathrm{L}=0.1 ; 0.01 ; 0.001$, respectively.

approximation works for the shell model, but, since the $O(1)$ energy flux is fixed by the forcing function, the growth of kinetic energy must be related to a relaxation time $\tau_{r} \rightarrow 0$ at $d=d_{c}$ and a corresponding small parameter. This result also shows that the phenomenon is very robust: all one needs is a point at which energy cascade changes its direction. The results of the shell model investigation will be published elsewhere [21]. The expression (47) is similar to the one obtained in a ground-breaking paper by Polyakov on the scale-invariance of strong interactions, where the multifractal scaling and the pdf were analytically derived for the first time [22]-[23]. In the review paper [23] Polyakov noticed that the exact result can be simply reproduced considering a cascade process with a heavy stream (particle) transformed into lighter streams at each step of the cascade (fission). Due to the relativistic effects the higher the energy of the particle, the smaller the angle of a cone, accessible to the fragments formed as a result of fission. Thus, the larger the number of a cascade step, the smaller is the fraction of space occupied by the particles [23]. The theory, presented in this paper describes many experimental observations. Still, understanding of the limits of validity of expression (29) is crucial for the final assessment of the theory. The relation (32) shows that (29) is consistent with the gaussian tails of the pdf. However, at the present stage we are unable to prove that (29) is the only expression leading to this result. The problem is that without experimental detection of at least some deviations from the the gaussian statistics of transverse velocity differences, one will not be able to understand the limits of validity of (29). Given the state-of-the-art of numerical simulations, this goal may not be that simple. 


\section{Acknowledgment}

I am grateful to A. Polyakov for most interesting and stimulating discussions. Help from K.R. Sreenivasan and M. Vergassola, who shared with me their sometimes unpublished experimental data, was most usefull. Recent numerical results of M. Jensen produced not only support of conclusions of this paper but also demonstrated a surprising power and importance of a shell model. I benefited a lot from conversations with B. Shraiman, P. Constantin, C. Doering, T. Gotoh and T. Kambe.

\section{References}

1. M. Cherkov, G. Falkovich, I. Kolokolov and V. Lebedev, Phys. Rev. E, 52, 4924 (1995)

2. K. Gawedzki and A. kupiainen, Phys. Rev. Lett., 75, 3834 (1995)

3. B. I. Shraiman and E. D. Siggia, C.R. Acad. Sci., 321, Serie II, 279 (1995)

4. L.Smith and V. Yakhot, J. Fluid. Mech. 274, 115 (1994)

5. L.Smith and V. Yakhot, Phys.Rev.Lett. 71, 352 (1993)

6. P. Tabeling and J. Paret, Phys. Fluids, 12, 3126 (1998)

7. G. Bofetta, A. Cellini, M. Vergassola, chao-dyn/9906016

8. U. Frisch, "Turbulence", Cambridge University Press, 1995

9. U. Frisch and J.D. Fournier, Phys. Rev. A, 17, 747 (1978)

10. V. Yakhot and S.A.Orszag, Phys.Rev.Lett. 57, 1722 (1986)

11. V. Yakhot, Phys. Rev.E, in press (1999)

12. A.M. Polyakov, Phys.Rev. E, 52, 6183 (1995)

13. L.D.Landau and E.M. Lifshitz, Fluid Mechanics, Pergamon Press, Oxford, 19814. A.S.Monin and A.M.Yaglom, "Statistical Fluid Mechanics" vol. 1, MIT Press, Cambridge, MA (1971)

15. Ya. G. Sinai and V. Yakhot, Phys.Rev.Lett., 63, 1962 (1989)

16. R.H.Kraichnan, Phys.Fluids. 10, 1417 (1967)

17. Yakhot Orszag, J. Sci. Comp., 1, 3 (1986)

18. V. Yakhot, Phyds. Rev. E., 55, 329 (1997) 
V. Yakhot, Phys. Rev.E, Phys. Rev. E, 57, 1737 (1998)

19. K.R. Sreenivasan, private communication

20. M. Jensen, private communication

21. M. Jensen and V. Yakhot, to be published

22. A.M. Polyakov, Sov. Phys. JETP 34, 1177 (1972)

23. A.M. Polyakov, "Scale Invariance of Strong Interactions and Its Application to LeptonHadron Reactions", preprint, Landau Institute for Theoretical Physics, 1971 